\title{
Anti-CD37 MMAE Antibody-drug Conjugate AGS67E
}

National Cancer Institute

\section{Source}

National Cancer Institute. Anti-CD37 MMAE Antibody-drug Conjugate AGS67E. NCI

Thesaurus. Code C119700.

An antibody-drug conjug ate (ADC) composed of AGS67C, a human anti-CD37

monoclonal antibody covalently linked, via reduced cysteines and a protease cleavable

linker, to monomethyl auristatin $\mathrm{E}$ (MMAE), an auristatin derivative and a potent

microtubule disrupting agent, with potential antineoplastic activity. Upon administration, the monoclonal antibody moiety of AGS67E binds to CD37 antigens on tumor B-cells and is rapidly internalized, thereby delivering MMAE intracellularly. Upon proteolytic cleavage, MMAE binds to tubulin and inhibits its polymerization, resulting in G2/M checkpoint arrest and apoptosis in CD37-expressing tumor cells. CD37, a transmembrane glycoprotein, is overexpressed in B-cell malignancies. 